\title{
Investigation of Continuum Breakdown in Hypersonic Flows Using a Hybrid DSMC-NS Algorithm
}

\author{
Thomas E. Schwartzentruber* \\ Department of Aerospace Engineering and Mechanics, University of Minnesota, Minneapolis, MN 55455 \\ and Iain D. Boyd ${ }^{\dagger}$ \\ Department of Aerospace Engineering, University of Michigan, Ann Arbor, MI 48109
}

\begin{abstract}
A new method of determining continuum breakdown in hypersonic flows that is part of a modular particlecontinuum numerical method is presented. The modular particle-continuum method loosely couples direct simulation Monte-Carlo and Navier-Stokes methods which operate in different regions, use different mesh densities, and are updated using different sized timesteps. The hybrid method includes overlapping regions where both particle and continuum methods are used. The new method of detecting continuum breakdown involves the direct comparison of particle and continuum solutions within such overlapping regions. Such a method of continuum breakdown detection eliminates the use of a breakdown parameter and associated empirical cutoff value used in the majority of prior hybrid research. Hybrid simulations of hypersonic flow over a cylinder using this new method are shown to accurately reproduce the flowfield properties and heating rates of full DSMC simulations. The new method of continuum breakdown detection is more computationally expensive than computing a breakdown parameter and is more sensitive to statistical scatter.
\end{abstract}

\section{Introduction}

$\mathrm{V}$ EHICLES traveling at hypersonic speeds and at high altitudes may generate flowfields that contain a mixture of near-continuum and nonequilibrium regions. Near-continuum flow is present in highly compressed regions downstream of strong shock waves, in stagnation regions, and in the far-wake of the vehicle. Nonequilibrium flow is present in the interiors of shock waves and thin hypersonic boundary layers, as well as in rapidly expanding regions in the near-wake of the vehicle. The continuum assumption inherent in the Navier-Stokes (NS) equations is known to break down for nonequilibrium flow and kinetic methods must be used to accurately model such regions. The most popular kinetic method for hypersonic flows is the direct simulation Monte-Carlo (DSMC) particle method. The cell size and timestep required for an accurate DSMC simulation are limited to the local mean-free-path $(\lambda)$ and mean-free-time $\left(\tau_{c}\right)$ respectively. Although the DSMC method provides an accurate model for both continuum and nonequilibrium flow, simulation of continuum regions where $\lambda$ and $\tau_{c}$ are typically very small becomes computationally intensive. However, it is precisely in such near-continuum regions where the NS equations provide an accurate physical model and can be solved efficiently (without such cell size and timestep restrictions) using methods from computational fluid dynamics (CFD). For this reason, significant research has been devoted to the development of hybrid particle-continuum numerical methods. The goal of such hybrid methods is to use efficient CFD methods to solve the NS equations where they are accurate and restrict use of the kinetic particle method to nonequilibrium regions only. Various hybrid methods applied to a range of applications are detailed in Refs. 1-7. A concise summary of this prior research as well as a complete description of the hybrid algorithm used for this article, called the modular particle-continuum (MPC) method, can be found in Ref. 8.

The major considerations involved in the development of a hybrid DSMC-NS method include information transfer between DSMC and NS regions (requiring the control of statistical scatter), the numerical cycle that dictates when to transfer information (the coupling cycle), and determining in which regions the DSMC method is required (continuum breakdown). Reference 9 presents a discussion of these considerations and Ref. 8 discusses these issues in context with the MPC method used to generate the hybrid results in this article. Typically, continuum breakdown is assumed

*Assistant Professor, Member AIAA. Email: schwartz@aem.umn.edu.

${ }^{\dagger}$ Professor, Associate Fellow AIAA. Email: iainboyd@umich.edu. 
when a locally computed continuum breakdown parameter exceeds an empirically-set cutoff value. Prior studies have suggested optimal cutoff values by comparing full DSMC and NS solutions to hypersonic flow problems. This article presents further research concerning continuum breakdown in hypersonic flows. In contrast to previous studies, in addition to comparing full DSMC and NS results, this article utilizes a hybrid DSMC-NS method (the MPC method) to study continuum breakdown and introduces a coupling method that eliminates the reliance on such an empirical breakdown parameter and cutoff value. The coupling algorithm as well as its advantages and disadvantages are detailed for the specific problem of hypersonic flow over a 2D cylinder. This simple, blunt-body geometry creates a complex flow containing virtually all of the flow features of interest. Such features include a strong bow shock, a stagnation region, a thin hypersonic boundary layer on the forebody of the cylinder, rapidly expanding rarefied flow, and a region of recirculating flow in the wake. In addition, such a flowfield contains regions of thermal non-equilibrium as well as near-continuum regions where the flow is completely in thermal equilibrium. The accuracy and computational efficiency of the MPC method using various continuum breakdown criteria is investigated in this article.

\section{Detecting Continuum Breakdown in Hypersonic Nonequilibrium Flows}

The continuum assumption inherent in the Navier-Stokes equations is that throughout the flowfield, the local distribution of molecular velocities is near equilibrium (a first-order perturbation to a Maxwellian distribution called a Chapman-Enskog distribution). This assumption directly leads to the closure models for heat and momentum transfer found in the NS equations. In two dimensions, the viscous stress tensor (momentum transfer) under Stokes' hypothesis is given by:

$$
\begin{aligned}
\tau_{x x} & =2 \mu\left[\frac{\partial u}{\partial x}-\frac{1}{3} \nabla \cdot \mathbf{V}\right], \\
\tau_{y y} & =2 \mu\left[\frac{\partial v}{\partial y}-\frac{1}{3} \nabla \cdot \mathbf{V}\right], \\
\tau_{x y} & =\tau_{y x}=\mu\left[\frac{\partial u}{\partial y}+\frac{\partial v}{\partial x}\right],
\end{aligned}
$$

where $\mathbf{V}(u, v, w)$ is the bulk fluid velocity vector. For both DSMC and NS simulations, the coefficient of viscosity, $\mu$, is modeled using the same power-law relationship given by:

$$
\mu=\mu_{r e f}\left(\frac{T}{T_{r e f}}\right)^{\omega}, \quad \mu_{r e f}=\frac{15 \sqrt{\pi m k T_{r e f}}}{2 \pi d_{r e f}^{2}(5-2 \omega)(7-2 \omega)} .
$$

This model is consistent with the variable hard sphere (VHS) collision model used in the DSMC method. All numerical results presented in this article are for diatomic nitrogen with a reference diameter of $d_{\text {ref }}=4.17 \times 10^{-10} \mathrm{~m}$ at a reference temperature, $T_{r e f}=273 \mathrm{~K}$. The power law exponent, $\omega$, is set equal to $0.75, \mathrm{~m}$ is the mass of an $\mathrm{N}_{2}$ molecule, and $k$ is the Boltzmann constant. The heat transfer closure model is given by Fourier's law in two dimensions:

$$
q_{x}=-\kappa \frac{\partial T}{\partial x} \text { and } q_{y}=-\kappa \frac{\partial T}{\partial y}
$$

where the thermal conductivity, $\kappa$, is determined from Eucken's relation

$$
\kappa=\mu\left(\frac{5}{2} C_{V}^{(t r)}+C_{V}^{(r o t)}\right)
$$

Here, $C_{V}^{(t r)}=\frac{3}{2} R_{\text {gas }}$ and $C_{V}^{(r o t)}=R_{\text {gas }}$ are the translational and rotational contributions to the specific heat of the gas at constant volume. Since the viscosity models are made consistent between DSMC and NS simulations in the continuum limit, the models for thermal conductivity are also consistent.

If the local velocity distributions are highly non-equilibrium, it is the above closure models (which rely on macroscopic velocity and temperature gradients) that become inaccurate. Such continuum breakdown can be investigated using full DSMC simulation results. If sufficient samples are collected during a DSMC simulation, local velocity distribution functions can be populated and compared to the corresponding Chapman-Enskog distributions assumed by the NS equations. Such analysis has recently been performed for hypersonic flow over a $2 \mathrm{D}$ cylinder. ${ }^{10}$ This study demonstrated that the resulting flowfield contains a mixture of near-continuum and nonequilibrium regions. Equivalently, using a full DSMC solution, the mass and momentum transfer between cells can be compared with that predicted 
by Fourier and Stokes' closure laws. ${ }^{11}$ Another interesting method of determining regions of continuum breakdown involves using DSMC particle information to compute entropy production. ${ }^{12}$ Locally, if entropy production is small the flow can be assumed to be in near-equilibrium. However, regions associated with large entropy production are highly nonequilibrium. Although these types of analysis are able to accurately assess continuum breakdown within a flowfield, such analysis requires a highly sampled, full DSMC simulation. Since the purpose of a hybrid DSMC-NS method is to avoid such a costly simulation, alternate methods of determining continuum breakdown are required for practical use within a hybrid algorithm.

The most widely used approach is to locally compute a measure of continuum breakdown (a breakdown parameter) that is a function of macroscopic continuum quantities and associated gradients. The parameter used by the MPC method in prior studies is the gradient-length Knudsen number, ${ }^{13,14}$

$$
K n_{G L-Q}=\frac{\lambda}{Q}|\nabla Q|
$$

In order to capture breakdown of all transport properties, a maximum is taken over the flow quantities $(Q)$ of interest: density $(\rho)$, temperature $(T)$, and velocity magnitude $(|V|)$,

$$
K n_{G L}=\max \left(K n_{G L-\rho}, K n_{G L-T}, K n_{G L-|V|}\right)
$$

A cutoff value $\left(B r_{\text {cutoff }}\right)$ is selected so that cells in which $K n_{G L}>B r_{\text {cutoff }}$ are labelled as DSMC cells and the remainder as NS cells, thus defining an interface between the two regions. The local mean-free-path is calculated using the macroscopic properties ${ }^{15}$ in each cell as

$$
\lambda=\frac{2(5-2 \omega)(7-2 \omega)}{15} \sqrt{\frac{m}{2 \pi k T}}\left(\frac{\mu}{\rho}\right),
$$

where $\mu$ is the local viscosity from Eq. 2 .

The gradient-length Knudsen number has a physical basis in that the temperature and velocity terms appear as higher-order terms of the Chapman-Enskog velocity distribution function. ${ }^{15}$ Thus large values of $K n_{G L}$ indicate significant departure from an equilibrium velocity distribution. Effectively, this parameter compares the gradient-length to the local mean-free-path. If the flow variable is undergoing a significant percentage change $\left(>B r_{c u t o f f}\right)$ over one mean-free-path, then the flow is likely in nonequilibrium. An optimal cutoff value of the breakdown parameter is determined empirically by comparing the difference between DSMC and NS solutions with the local value of the breakdown parameter. A cutoff value is then chosen that best detects regions of large discrepancy for as many flowfields as possible. Previous studies have recommended ${ }^{13,14}$ a cutoff value of $B r_{\text {cutoff }}=0.05$. These studies investigated continuum breakdown in the forebody of a blunt-body flow and for flow over a wedge and hollow cylinder-flare geometry by comparing full DSMC and NS solutions. One drawback of determining the optimal cutoff value in this manner is that nonequilibrium effects next to the surface can 'shift' significant portions of the flowfield leading to large regions of discrepancy between DSMC and NS. This is especially important in the wake region of a blunt-body flow.

As an example, Fig. 1 plots full DSMC and full NS solutions for the translational temperature field resulting from Mach 12 flow over an $8 \mathrm{~cm}$ diameter cylinder at approximately $70 \mathrm{~km}$ altitude. The specific free-stream density and temperature are $\rho=7.48 \times 10^{-5} \mathrm{~kg} / \mathrm{m}^{3}$, and $T=217.45 \mathrm{~K}$. Assuming the gas is comprised only of $\mathrm{N}_{2}$, these conditions correspond to a number density, $n=1.61 \times 10^{21} 1 / \mathrm{m}^{3}$, and pressure, $p=4.83 \mathrm{~Pa}$. The free-stream velocity is set to $3608 \mathrm{~m} / \mathrm{s}$ resulting in Mach 12 flow and the cylinder wall temperature is assumed constant at $1000 \mathrm{~K}$. The NS simulation assumes no slip conditions and the DSMC simulation assumes diffuse reflection and full thermal accommodation at the surface. It is evident from Fig. 1 that DSMC predicts a thicker shock wave with an overshoot in the translational temperature. The temperature predicted by both DSMC and NS simulations near the stagnation region and forebody surface is in very good agreement. However, as the flow expands about the cylinder, the temperature predicted by DSMC is seen to be uniformly higher than that predicted by NS in the wake. The largest discrepancy is at the cylinder surface in the wake region where significant velocity slip and temperature jump occurs due to the rapidly expanding rarefied flow. Figure 2 compares the gradient-length Knudsen number with the discrepancy between DSMC and NS solutions along cuts C1 through the forebody, and C2 through the wake. In both Figs. 2(a) and 2(b), the gradient-length Knudsen number for density, velocity magnitude, and translational temperature is plotted in the upper portions of Fig. 2. The percent error between the NS solution and the full DSMC solution for each variable of interest is represented by

$$
E_{Q}=\frac{\left|Q_{D S M C}-Q_{N S}\right|}{Q_{D S M C}}
$$




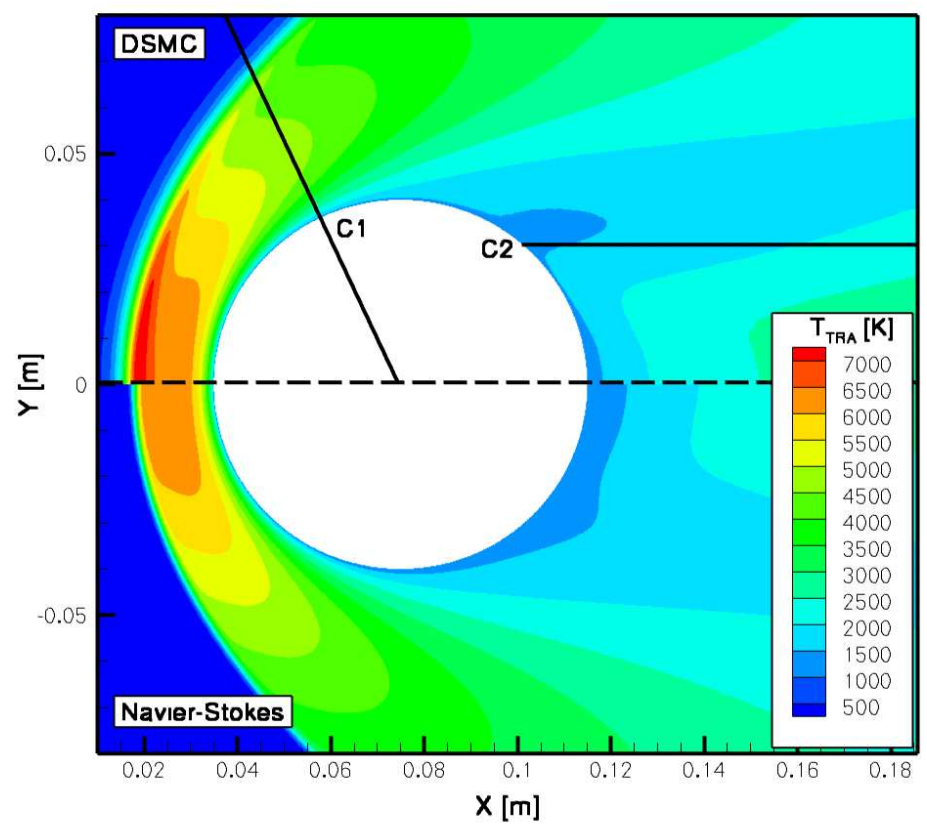

Figure 1. DSMC and NS solutions for Mach 12 flow over a 2D cylinder geometry.

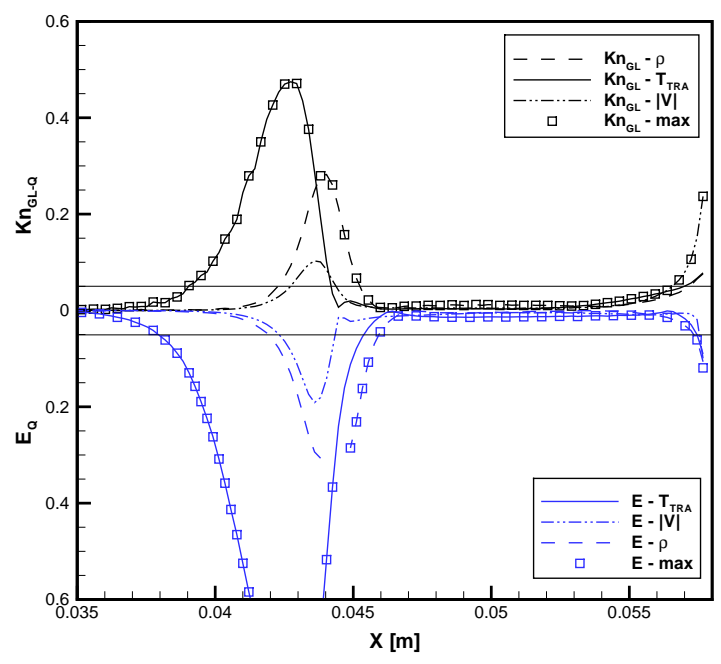

(a) Continuum breakdown in the forebody flow (cut C1).

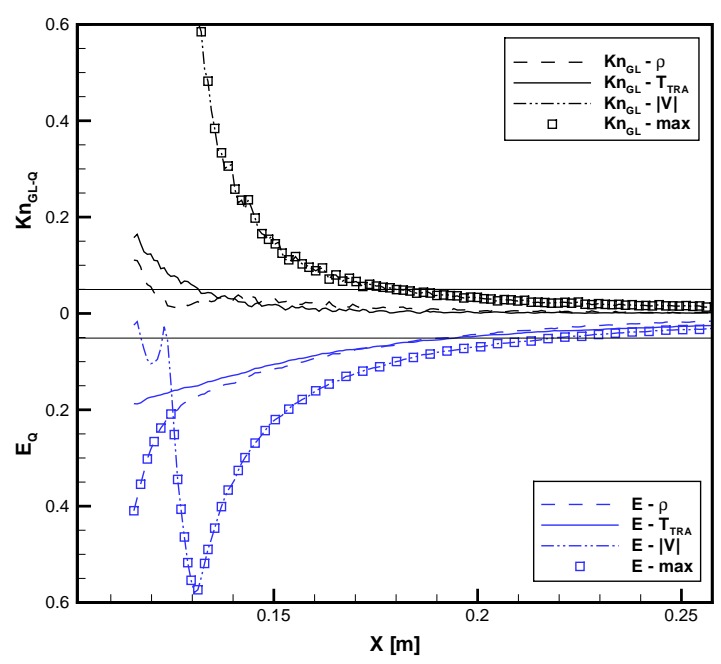

(b) Continuum breakdown in the wake flow (cut C2).

Figure 2. Continuum breakdown and discrepancy between DSMC and NS solutions. 
and is plotted in the bottom portions of Fig. 2. The maximum of $K n_{G L-Q}$ and $E_{Q}$ is highlighted by the symbols in Fig. 2. It is important to note, that for such a comparison to be useful the continuum breakdown parameter must be computed using the DSMC solution and not the NS solution. Of interest is how the gradient-lengths in the correct physical solution compare with the local mean-free-path. Nonequilibrium flow regions should exhibit significant discrepancy between DSMC and NS solutions.

This first observation to note from Figs. 2(a) and 2(b) is that the $K n_{G L}$ parameter predicts regions of large discrepancy very well and reasonably predicts the magnitude of the discrepancy. This result is consistent with previous research. ${ }^{10}$ For reference, in Figs. 2(a) and 2(b), the recommended cutoff value $\left(B r_{\text {cutoff }}=0.05\right)$ is represented with a horizontal line, as is the line of $5 \%$ error $\left(E_{Q}=0.05\right)$. Along cut $C 1$ through the forebody flow, it is evident that the discrepancy in translational temperature is the largest. This is due to the temperature overshoot predicted by DSMC and the fact that this temperature increase occurs significantly upstream compared to the NS result. Clearly, the $K n_{G L}$ parameter does a very good job in predicting the amount of discrepancy for each variable at the correct position within the shock. However, close inspection of the shock region shows that a cutoff value of 0.05 is not quite sufficient. Specifically, if near-continuum flow is assumed for $K n_{G L}<0.05$, there may be regions in which the error is greater than 5\%. A discrepancy of this magnitude is especially detrimental for the post shock state since the error is convected towards the surface and directly influences the heating rates to the vehicle. Conversely, next to the cylinder surface, the $K n_{G L-|V|}$ parameter is seen to be conservative in Fig. 2(a). That is, the maximum error is quite low despite a value of $K n_{G L-|V|}>0.05$ signifying continuum breakdown. The results along cut $\mathrm{C} 2$ through the wake region are plotted in Fig. 2(b). Again, the $K n_{G L}$ parameter succeeds in predicting the discrepancy between DSMC and NS very well for each flow variable. The temperature jump leads to the largest error next to the surface and the discrepancy in velocity is largest throughout the remainder of the wake. Inspection of Fig. 2(b) reveals that when $K n_{G L}<0.05$ (signifying near-continuum flow), the discrepancy is larger than $5 \%$ for a significant portion of the wake. This suggests that setting $B r_{\text {cutoff }}=0.05$ is not stringent enough in the wake region and a very low cutoff value would be required in order to keep the discrepancy below 5\%. As mentioned above, this discrepancy (which persists through the wake region) is a result of the fact that the velocity field is effectively 'shifted' between DSMC and NS solutions due to velocity slip next to the surface. For this reason, empirical determination of the cutoff value for continuum breakdown by comparing DSMC and NS solutions has limitations.

Overall, the gradient-length Knudsen number is demonstrated to be an accurate and reliable method of identifying regions of continuum breakdown in a blunt-body flowfield. However, the recommended cutoff value $\left(B r_{\text {cutoff }}=0.05\right)$ is seen to be insufficient to capture the entire shock wave and is seen to be conservative in the boundary layer. The cutoff value of 0.05 appears to be insufficient in the wake region, however, this is due to the 'shift' between DSMC and NS solutions. When actually incorporated within the MPC method, the results presented in section IV will show this cutoff to be conservative in the wake. It is important to note that using a conservative breakdown parameter within a hybrid DSMC-NS algorithm affects the efficiency of the hybrid method and not the accuracy of the solution. If the parameter is conservative, the hybrid method is simply simulating a larger-than-necessary region with the DSMC method. However, previous research has determined that the full width of the shock must be modeled with DSMC in order to obtain an accurate hybrid solution. Such research added a condition of thermal equilibrium to the $K n_{G L}$ breakdown parameter to ensure the entire shock wave is modeled using DSMC. ${ }^{8,10}$ This is implemented by calculating the difference between translational and rotational temperatures in the hybrid solution as

$$
E_{\text {thermal }}=\frac{T_{T R A}-T_{R O T}}{T_{R O T}} .
$$

If the value of $E_{\text {thermal }}>0.01$, then the region is considered to be in nonequilibrium and the DSMC method is used. Note that this condition is only met in compression thermal nonequilibrium regions where $T_{T R A}>T_{R O T}$ such as in the vicinity of shock waves.

An advantage of studying continuum breakdown using a hybrid DSMC-NS method is that it is possible to simply test different breakdown parameters and observe the accuracy of the hybrid result. In addition, the MPC method contains overlapping regions in which both DSMC and NS methods are used during the simulation. This allows for local comparison between the DSMC and NS solutions during the simulation. If the solutions agree in the overlap regions, then the DSMC-NS interfaces are properly positioned. If the NS method is unable to reproduce DSMC results inside the overlap region, then the interfaces may be located in nonequilibrium regions and require an updated position. Such a coupling cycle will be described and tested in the remainder of this article. 


\section{Modular Particle-Continuum Numerical Method}

\section{A. Problem setup and information transfer}

The modular particle-continuum (MPC) method used in this article was initially developed and tested on normal shock waves ${ }^{16}$ and later on 2D flow over a cylinder. ${ }^{8}$ Recently it has successfully been applied to more complex axisymmetric flows about a planetary probe geometry and a hollow cylinder flare geometry. ${ }^{17}$ Within the MPC numerical algorithm, particle regions are simulated using MONACO, ${ }^{18}$ a general, cell-based implementation of the DSMC method. ${ }^{15}$ The variable hard sphere (VHS) collision model is employed which results in the macroscopic viscosity model detailed in Eq. 2. MONACO employs the variable rotational energy exchange probability model of Boyd ${ }^{19}$ where the reference temperature for rotational energy exchange is specified as $91.5 \mathrm{~K}$ and the maximum rotational collision number as 18.1. Energy transfer to vibrational modes is not considered. Continuum regions are simulated using the LeMANS code. ${ }^{20}$ In continuum regions, it is assumed that rotational and translational energy modes can be described by a single temperature $T$ and the vibrational energy mode is not considered. The resulting governing equations are the wellknown, 2D laminar, compressible, Navier-Stokes (NS) equations. The viscosity in the NS solver is modeled using Eq. 2 in order to match exactly the viscosity model used in DSMC and no-slip conditions are applied on all surfaces. Specific details of the LeMANS code are contained in Ref. 20.

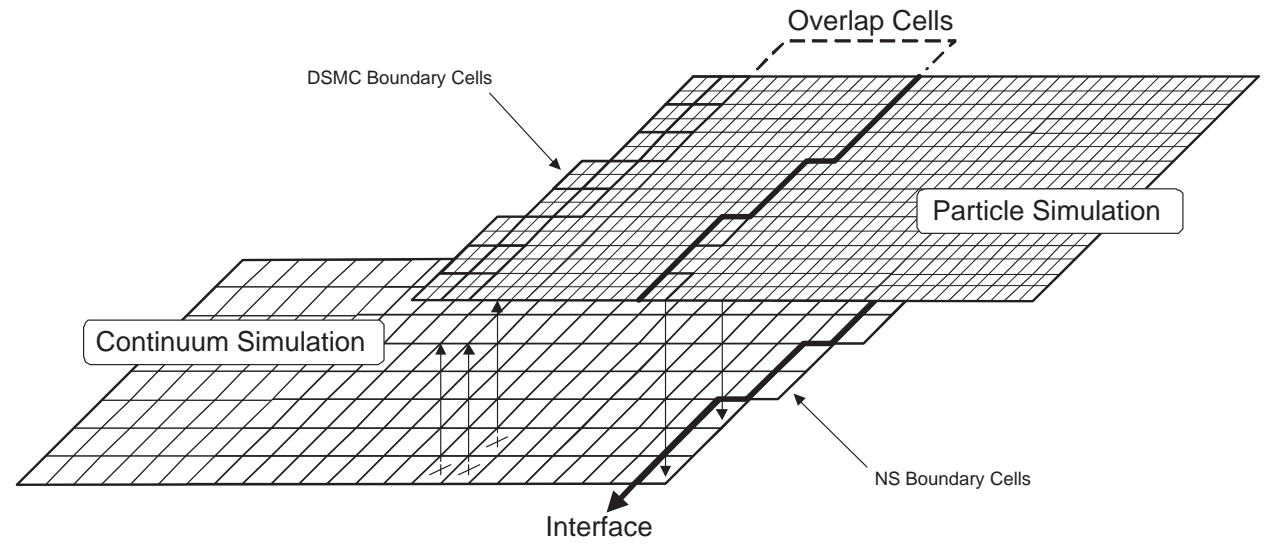

Figure 3. Information transfer for the MPC method.

The MPC method begins with a NS solution obtained on a mesh designed to solve the NS equations. An estimate of local continuum breakdown is provided by the maximum gradient-length Knudsen number (Eq. 6). Cells in nonequilibrium regions are then labeled as DSMC cells. In order to create an overlap region where both methods are used, the DSMC regions are then extended further into continuum regions by a number $\left(n_{\text {overlap }}\right)$ of cells. For the $2 \mathrm{D}$ cylinder simulations studied in this article a value of $n_{\text {overlap }}=4$ is used. Extended DSMC regions are further surrounded by two additional DSMC boundary cells and NS regions are surrounded by one additional NS boundary cell. Now that all regions and boundaries have been initialized, the mesh inside DSMC regions is refined to the local value of $\lambda^{21}$ Initially particles are generated in all DSMC cells (using a Chapman-Enskog velocity distribution ${ }^{22}$ ) such that they are consistent with the initial NS solution. Prior to each DSMC simulation timestep, all particles within DSMC boundary cells are first deleted and then regenerated to maintain the desired boundary conditions, consistent with the corresponding NS solution. As the DSMC regions iterate and particles collide with each other and with surfaces, the DSMC solution proceeds away from the initial continuum NS solution towards the correct nonequilibrium solution including thermal nonequilibrium. As this progression and relaxation occurs, DSMC and NS regions are loosely coupled and interface locations are periodically updated throughout the simulation. The precise manner in which continuum breakdown is determined and the interfaces updated will be discussed in detail shortly. Prior to the NS portions of the hybrid numerical cycle, the macroscopic state variables are set in each NS boundary cell using a sub-relaxation average $\mathrm{e}^{23}$ of the molecular properties in each corresponding DSMC cell. The sub-relaxation average is able to track macroscopic variations in the DSMC solution with low statistical scatter. A schematic of the overlap regions, DSMC and NS boundary cells, and information transfer process is depicted in Fig. 3. 


\section{B. Revised MPC coupling procedures}

The loosely coupled numerical cycle employed in the MPC method that determines when to transfer information between DSMC and NS regions can be summarized as follows:

1. Use the initial NS solution and the criteria $\left(K n_{G L}>B r_{\text {cutoff }}\right)$ to setup the initial DSMC and NS domains. Refine DSMC regions to $\lambda$ and generate particles throughout the entire DSMC domain. The hybrid simulation is now initialized.

2. Allow DSMC regions to progress and relax with the current boundary conditions. Periodically compute a measure of continuum breakdown $\left(P_{B r}\right)$ based on the new hybrid-DSMC solution using one of the following methods:

(i) $P_{B r}=\max \left(K n_{G L}, E_{\text {thermal }}\right) \quad P_{B r}>B r_{\text {cutoff }}$ ?

(ii) $P_{B r}=E_{\text {thermal }} \quad P_{B r}>0.01$ ?

(iii) $P_{B r}=E_{Q} \quad P_{B r}>\varepsilon$ ? (tolerance $\varepsilon=3 \%$ )

Computing $E_{Q}$ requires converging NS regions for comparison with DSMC.

a) Set NS boundary conditions using new DSMC solution.

b) Significantly converge the NS regions.

c) Compute continuum breakdown parameter: $P_{B r}=E_{Q}$

d) Delete temporary (and possibly inaccurate) NS solution and revert to previous stored NS solution.

Proceed to update DSMC-NS interfaces based on the continuum breakdown result of method (i), (ii), or (iii).

3. After the DSMC solution and interfaces stop changing, use the current DSMC solution to set the NS boundary conditions. Significantly converge the NS region.

IF the new NS solution changes the state in any DSMC boundary cell $\longrightarrow$ return to 2 .

ELSE , if the new NS solution does not change the state in any DSMC boundary cell $\longrightarrow$ continue to 4 .

4. Since the NS region is converged and the DSMC solution is no longer changing, the interfaces will no longer change and steady-state has been reached. Lock the interfaces, remove the overlap regions, and cycle both the DSMC and NS methods (coupling occasionally) until the DSMC scatter and NS residual fall below threshold values.

All details of the above MPC numerical cycle are completely described in Ref. 8. Prior MPC research employed method (i) for the determination of continuum breakdown. This article investigates methods (ii) and (iii). It is important to note that for methods (ii) and (iii), the $K n_{G L}$ parameter must still be used at the start of the simulation (step 1) to identify likely areas of continuum breakdown. The important aspect of methods (ii) and (iii) is that the evolution and final steady-state position of the DSMC-NS interfaces does not rely on comparing $K n_{G L}$ to an empirically-set cutoff value. Rather, the overlap regions (shown in Fig. 3) allow for the direct comparison of NS and DSMC solutions and this information is used to adjust the interface locations accordingly. Method (ii) results from the observation that regions of nonequilibrium flow tend to correspond to regions of compression thermal nonequilibrium. Thus, in method (ii) the breakdown parameter $P_{B r}$ is determined solely by the difference between translational and rotational temperatures (Eq. 9). Method (iii) directly compares the macroscopic properties obtained by DSMC and NS methods in the overlap region. As discussed above, the overlap regions are intended to be near-equilibrium regions where NS and DSMC methods are both accurate models and should produce the same solution. Thus, within the overlap regions, if the difference between DSMC and NS solutions is greater than a small tolerance $(\varepsilon>3 \%)$, then the interface locations need to be adjusted. Conversely if the NS solution agrees with the DSMC solution, then the interface locations are adequately positioned. The tolerance for such a comparison can in theory be made vanishingly small for higher accuracy and therefore is not the same as an empirical cutoff value. As will be shown in the next section, one of the practical disadvantages of method (iii) is that the tolerance is limited by statistical scatter in the DSMC solution. 


\section{Progression of solution using revised continuum breakdown criteria}

The progression of an MPC simulation using method (iii) for detecting continuum breakdown is shown in Figs. 4 and 5 in the forebody and wake regions respectively. The new method is tested for both Mach 6 and Mach 12 flow over the cylinder geometry shown in Fig. 1 at the free-stream temperature and density listed previously in section II. The progression of an MPC solution is presented for the Mach 6 flow and only the results of the MPC simulations will be shown for the Mach 12 flow in section IV. The temperature profiles resulting from both full NS and full DSMC simulations of Mach 6 flow along cut $\mathrm{C} 1$ through the forebody flow are plotted in Fig. 4(a). The MPC solution is thus initialized to the full NS solution and during the MPC cycle, must progress towards the full DSMC simulation with a high degree of accuracy. In order to initially setup DSMC and NS domains (during step 1), a high cutoff value $\left(B r_{\text {cutoff }}>0.1\right)$ is used. This ensures that only highly nonequilibrium regions are initialized as DSMC regions. The initial interface locations and overlap regions are shown in Fig. 4(a). To begin the simulation, only a very thin region surrounding the steep continuum NS shock and a thin region next to the surface are considered nonequilibrium and initialized as pure DSMC cells. The overlap regions are then extended by a number of cells $\left(n_{\text {overlap }}=4\right)$ in each direction. Clearly the correct shock profile is much thicker than the initial NS shock profile and the DSMC region must enlarge as the MPC simulation continues. Figures 4(b) through 4(d) show the progression of the MPC solution in the vicinity of the bow shock wave. As the solution in the DSMC region progresses (step 2), the translational temperature profile begins to move upstream towards the correct DSMC profile. Fig. 4(b) depicts the MPC solution when the interface locations are updated for the first time. In order to determine if the interfaces are properly positioned, first the new DSMC solution (inside the shock) is used to update the boundary conditions for the NS regions. Next, the NS regions are significantly converged and the resulting profile in NS regions is shown by the dash-dotted line in Fig. 4(b). Clearly, despite using the updated boundary conditions from the DSMC regions, the NS solution differs significantly from the DSMC profile inside the overlap regions. In this case $E_{Q}$ will be large and cause cells in the overlap region to be converted to pure DSMC cells. After re-creating new overlap regions of $n_{\text {overlap }}=4$ cells, this results in the enlarged DSMC region seen in Fig. 4(c). It is important to note that the converged NS solution shown in Fig. 4(b) is only a temporary NS solution used for the sole purpose of comparison with DSMC inside overlap regions and is immediately discarded after $E_{Q}$ is computed. Next, the solution in the enlarged DSMC domain is allowed to progress further and the temperature profile at a later point in the simulation is shown in Fig. 4(c). Again, in order to determine if the interfaces are properly positioned, this updated DSMC solution is used to provide new boundary conditions for NS regions. In Fig. 4(c), the converged NS profile agrees very well with the DSMC temperature profile in the post-shock overlap region. In the post-shock overlap cells, the value of $P_{B r}=E_{Q}<\varepsilon$ (signifying near-equilibrium flow) and the post-shock interface will remain where it is. However, in the overlap region upstream of the shock wave, the NS equations are unable to reproduce the temperature profile computed by the DSMC method and the DSMC-NS interface will thus move further upstream. At a later time during the MPC cycle, the DSMC region has enlarged to encompass the entire temperature-shock profile as seen in Fig. 4(d). At this point, the interfaces are determined to lie in near-equilibrium regions and the MPC cycle advances to step 3. Figure 4(d) also plots the translational and rotational temperature profiles obtained by full DSMC simulation. The MPC solution is seen to have progressed into excellent agreement for both translational and rotational temperatures. Although not shown in Figs. 4(b) and 4(c), as the solution in the DSMC region progresses, thermal non-equilibrium also develops. Method (ii) monitors any thermal non-equilibrium within the overlap regions $\left(P_{B r}=E_{\text {thermal }}\right)$ and uses this information only to update the interface locations.

The progression of the MPC density profile for the same Mach 6 flow in the wake (along cut C2) is plotted in Figs. 5(a) through 5(d). The initial NS solution, full DSMC solution, and the initial interface locations are shown in Fig. 5(a). After the DSMC solution progresses and the interface locations are evaluated for the first time, the DSMC and NS solutions inside the overlap region differ noticeably as seen in Fig. 5(b). This results in a large value of $E_{Q}$ which signifies that the overlap regions may still lie in nonequilibrium flow and the DSMC region is enlarged. A similar result is seen for the next interface update in Fig. 5(c). However, as shown in Fig. 5(d), at some point the agreement in the overlap region becomes quite good and the interfaces cease to move. Unlike in the forebody, the statistical scatter in the DSMC profile is now evident. Although still distinguishable, the statistical scatter is becoming comparable to the discrepancy between DSMC and NS $\left(E_{Q}\right)$ in the overlap. Recall that the DSMC profiles result from the sub-relaxation average of DSMC particle information which is able to control the statistical scatter a great deal. ${ }^{16}$ However, a certain level of scatter is still present especially in highly rarefied regions such as the near wake. This scatter in macroscopic properties prevents setting a vanishing tolerance for $E_{Q}$. Although still robust for this flow, the tolerance must remain above $2-3 \%$ for the flows under consideration in order to reliably distinguish the true discrepancy between DSMC and NS solutions and statistical scatter. 


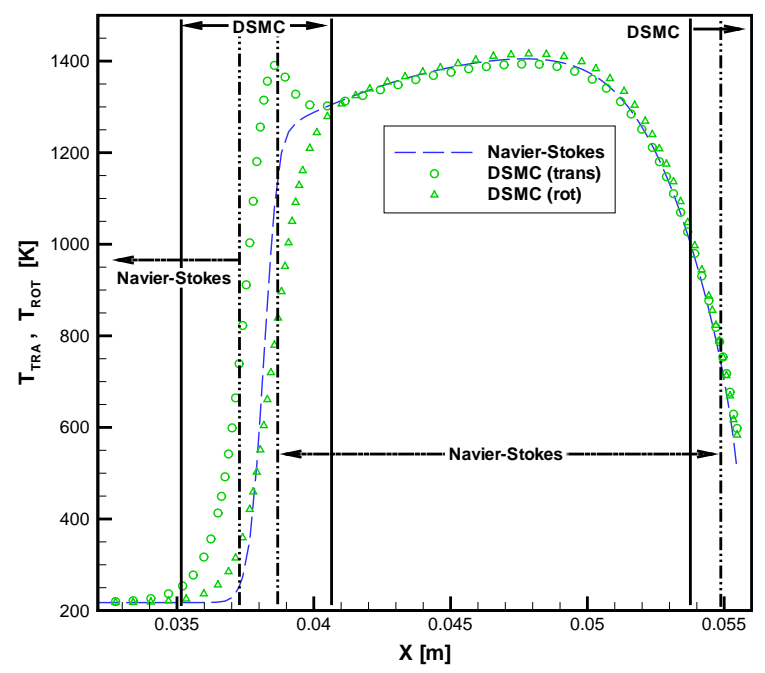

(a) Full DSMC and NS solutions.

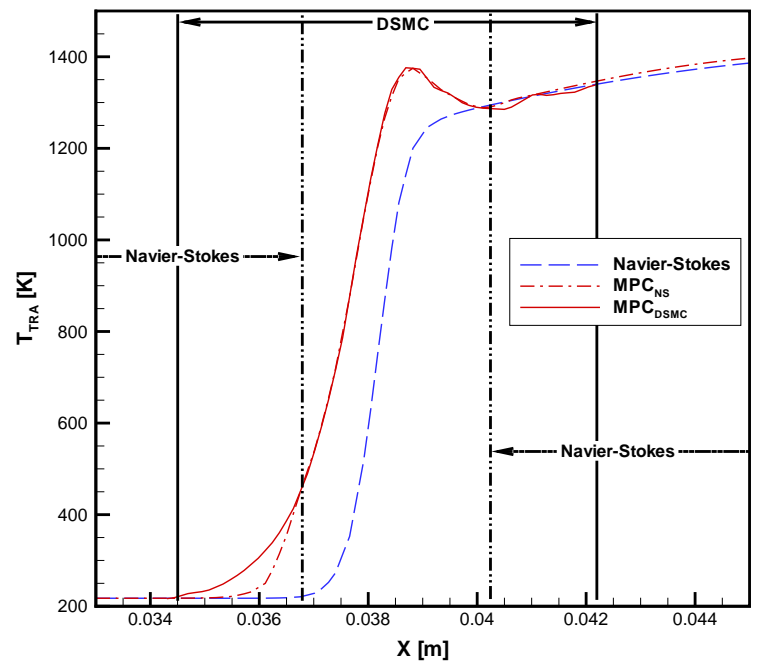

(c) MPC temperature profiles during second interface update.

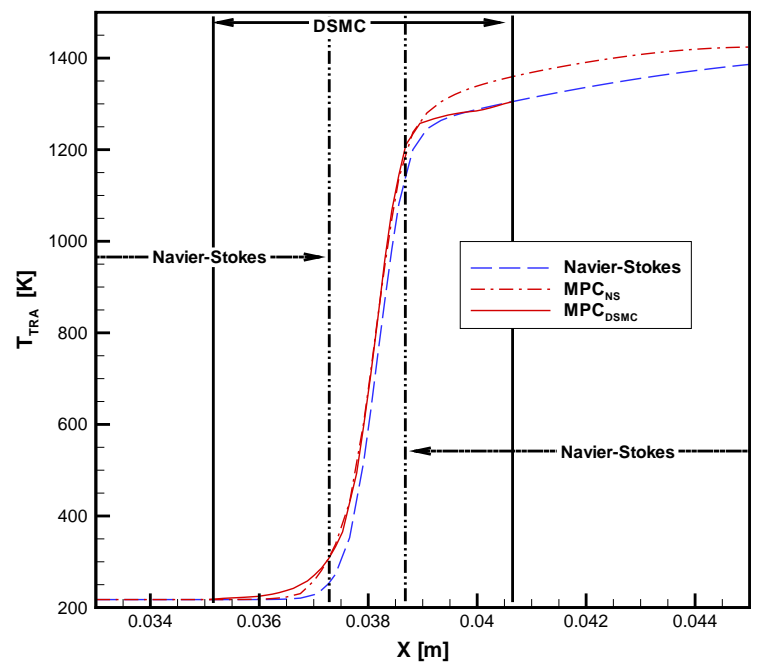

(b) MPC temperature profiles during first interface update.

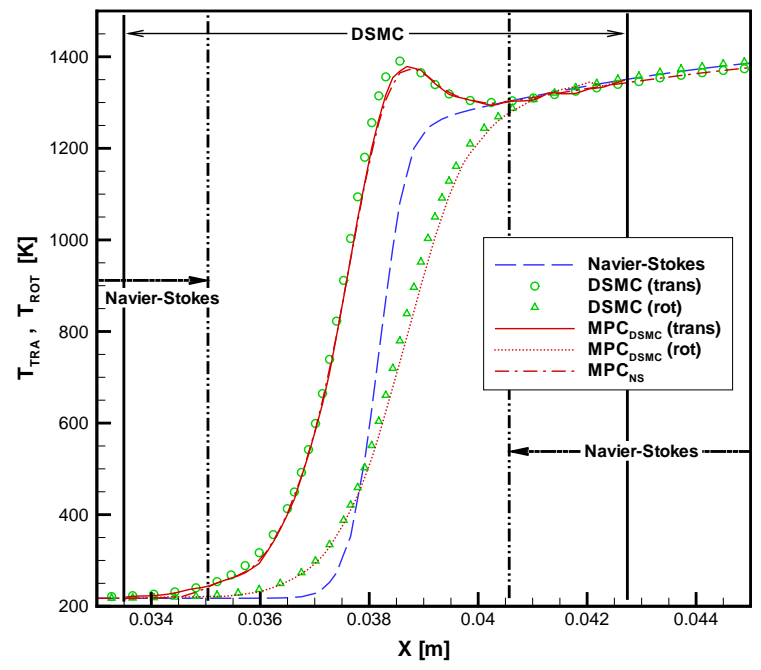

(d) MPC temperature profiles during final interface update.

Figure 4. Progression of MPC forebody temperature profile utilizing overlap regions to determine interface locations. 


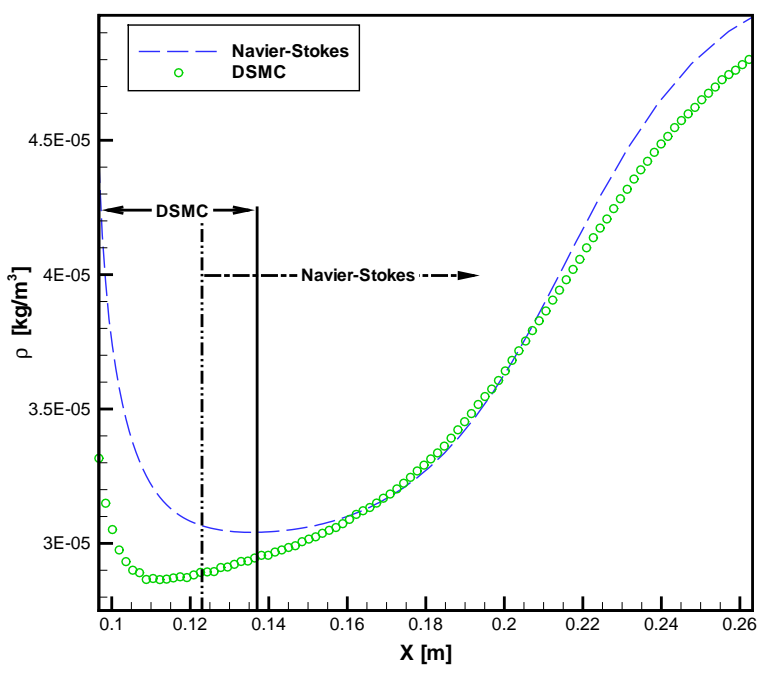

(a) Full DSMC and NS solutions.

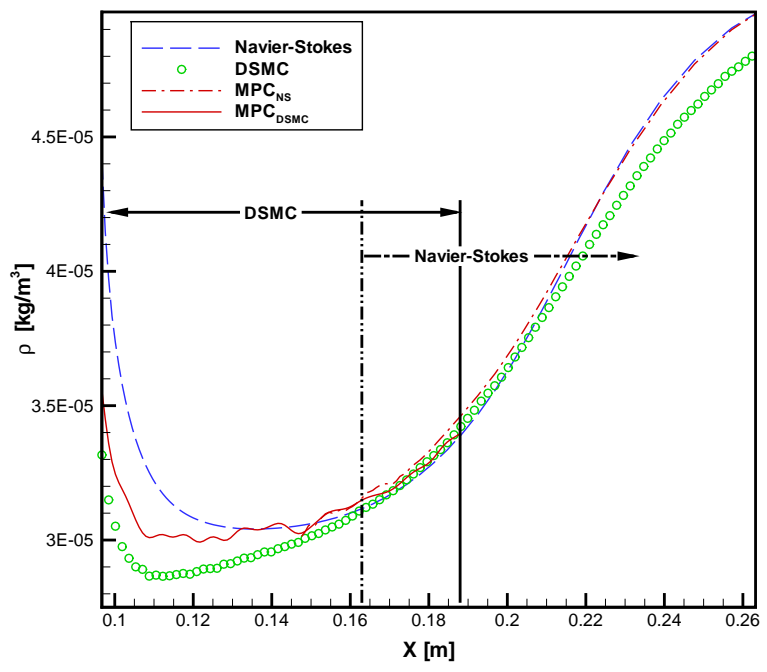

(c) MPC density profiles during second interface update.

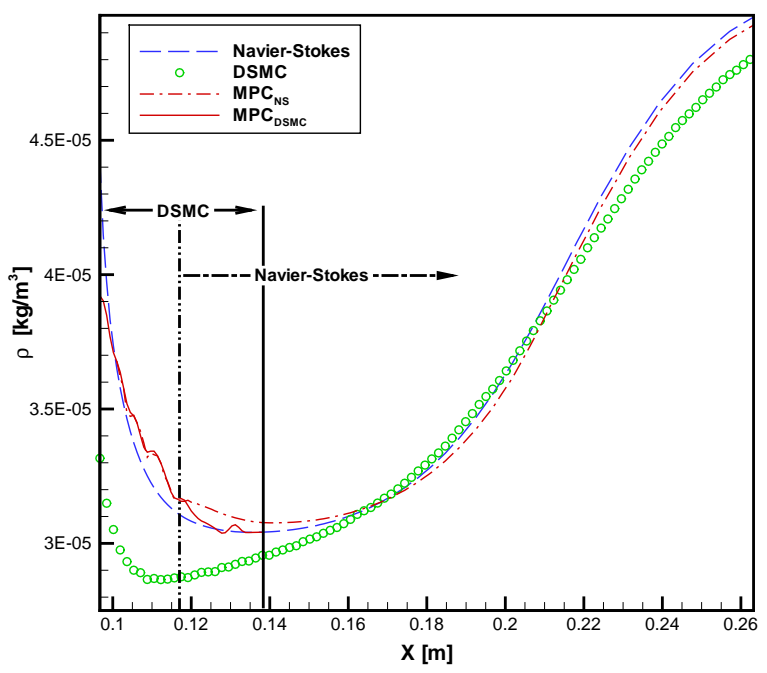

(b) MPC density profiles during first interface update.

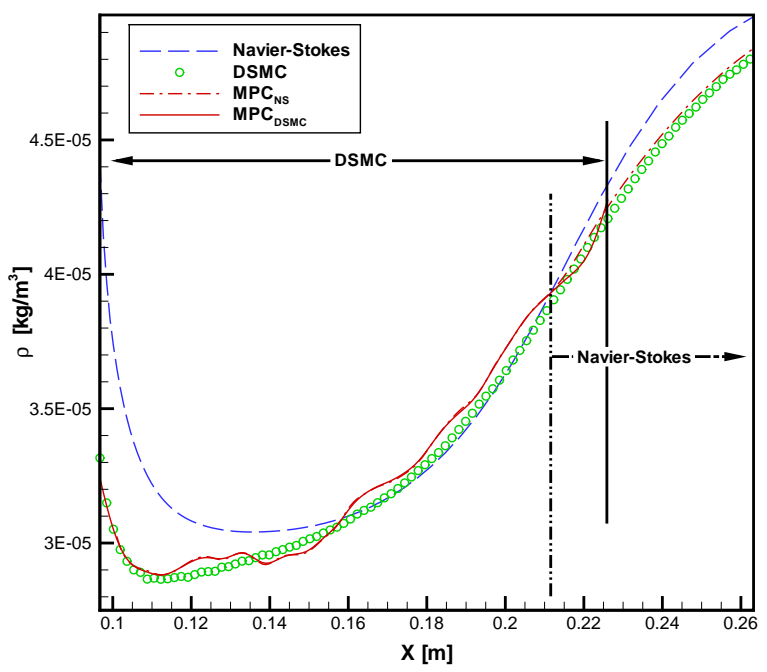

(d) MPC density profiles during final interface update.

Figure 5. Progression of MPC wake density profile utilizing overlap regions to determine interface locations. 


\section{Accuracy and Efficiency of MPC results}

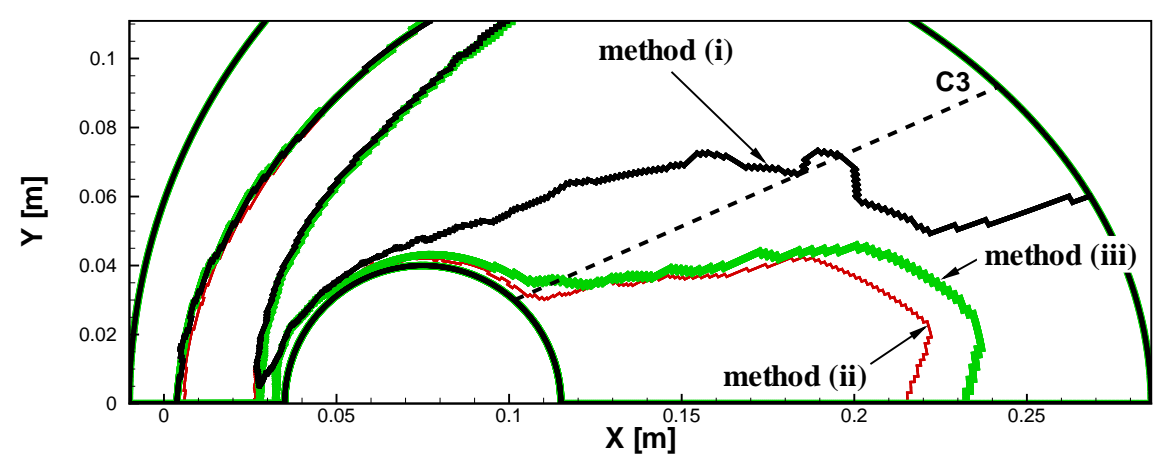

Figure 6. Final DSMC-NS interface locations corresponding to various methods of continuum breakdown detection.

The modified MPC algorithm, using methods (ii) and (iii) to determine continuum breakdown is used to simulate the Mach 12 flow shown earlier in Fig. 1. The new MPC results are compared with full NS, full DSMC, and prior MPC results that use the gradient-length Knudsen number (method (i)) to detect continuum breakdown. Figure 6 shows the final interface locations for the Mach 12 simulation using each of the three methods of determining continuum breakdown. All methods capture the bow shock effectively. Using the gradient-length Knudsen number (method (i)) is the most conservative both in the forebody where shock and stagnation DSMC regions merge, and in the wake where a significant portion of the flow is simulated using DSMC. Using method (ii), where continuum breakdown is based only on $E_{\text {thermal }}$ (compressive thermal nonequilibrium), DSMC regions enclose the entire bow shock and the recompression region in the near-wake flow. However, using method (ii), the forebody boundary layer is not considered to be in nonequilibrium and is therefore simulated using the NS equations. Compared with method (i), using method (iii) for detecting continuum breakdown is seen to use DSMC in a narrower region next to the forebody surface and significantly less DSMC in the wake. Since method (iii) ensures that DSMC and NS solutions agree in all overlap regions, this signifies that method (i) (with $K n_{G L}>0.05$ as the cutoff value) is conservative next to forebody surface and in much of the wake region. The accuracy of each simulation is now determined by comparison with full DSMC results for both heating rates on the cylinder surface and for flow properties along cut $\mathrm{C} 3$ seen in Fig. 6.

The heating rates predicted by the MPC algorithm using methods (ii) and (iii) for detection of continuum breakdown are plotted in Fig. 7 where they are compared with full NS and full DSMC results. Heating rates for the for the MPC results using method (i) have been previously verified to agree well with full DSMC results ${ }^{10}$ and are not plotted. In Fig. 7, the MPC results using method (iii) agree very well with DSMC over the entire cylinder surface. When continuum breakdown is detected using only $E_{\text {thermal }}$ (method (ii)), the MPC method reproduces NS results in the forebody (since DSMC in not used next to the surface) and transitions into good agreement in the wake where the DSMC method is used next to the surface. Thus relying on thermal nonequilibrium alone for the determination of continuum breakdown may not be adequate to reproduce full DSMC results in some regions. However, by ensuring that all DSMC and NS macroscopic properties agree in all overlap regions (method (iii)), the MPC method is able to accurately reproduce the heating rates predicted by DSMC. The simulation results plotted in Fig. 7 suggest that using $K n_{G L}>0.05$ (method (i)), is conservative in both the forebody boundary layer and wake regions since the modified MPC algorithm (using method (iii)) is able to successfully reproduce full DSMC heating rates despite simulating a smaller portion of the flowfield with DSMC.

The density and x-velocity profiles extracted from each solution along cut C3 in Fig. 6 are compared to full NS and DSMC solutions in Fig. 8. The vertical lines depict the final DSMC-NS interface locations resulting from the three different methods of detecting continuum breakdown. When continuum breakdown is determined by thermal nonequilibrium only (method (ii)), a very thin region next to the surface along cut C3 is simulated using DSMC. Method (iii) results in a slightly wider DSMC region near the surface and method (i) is seen to use DSMC for a large portion of the wake. Comparing NS and DSMC x-velocity profiles, it is evident that the properties in the far wake are 'shifted' due to nonequilibrium effects generated near the surface of the cylinder. Thus, although there appears to be a discrepancy between DSMC and NS solutions for a large portion of the wake, this region is a nearequilibrium region where the NS equations provide an accurate model if provided with the proper boundary conditions. 


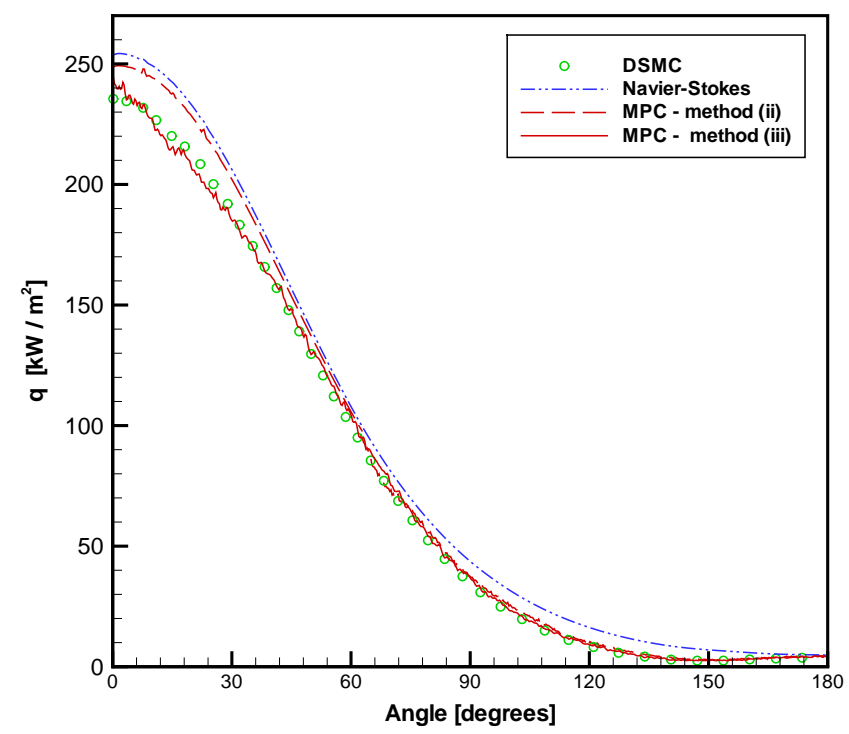

Figure 7. Heating rates on cylinder surface resulting from DSMC, NS, and MPC simulations.
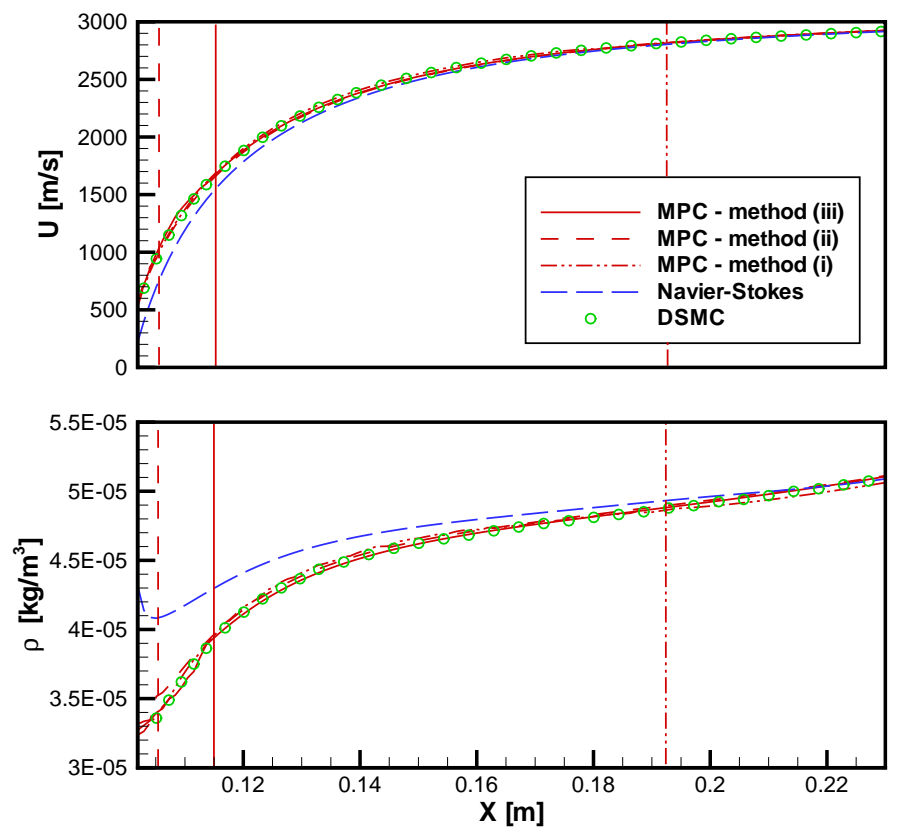

Figure 8. X-velocity and density profiles in the cylinder wake resulting from DSMC, NS, and MPC simulations. 
Figure 8 clearly shows that if the slip conditions next to the surface are passed as new boundary conditions to the NS region, the NS equations are able to shift the solution and reproduce full DSMC results. The results from method (iii) agree very well with those of the conservative method (i) and full DSMC results. The profiles resulting from method (ii) are also in surprisingly good agreement despite using DSMC in such a narrow region next to the surface. However, for this case a noticeable amount of error is evident in the density next to the surface. Thus method (ii), which uses only thermal nonequilibrium information to assess continuum breakdown, is able to adequately capture the bow shock and also performs well in the re-compression region in the wake of the cylinder. However, as the above results demonstrate, detecting compression thermal non-equilibrium alone does not guarantee that DSMC is used in all regions of continuum breakdown. Although method (ii) is a simple and efficient procedure it should be used as an added condition able to accurately determine continuum breakdown in the vicinity of shock waves.

For the cylinder simulations presented in this study, previous research has shown that the MPC method, using the gradient-length Knudsen number to detect continuum breakdown (method (i)), is able to reproduce full DSMC results with a high level of accuracy 1.6 to 3.3 times faster than full DSMC simulations. ${ }^{21}$ The revised MPC algorithm (method (iii)) is shown capable of accurately reproducing full DSMC results without relying on such a breakdown parameter and empirical cutoff value. Since this revised method which ensures that DSMC and NS solutions agree in all overlap regions is less conservative than the original criteria $\left(K n_{G L}<0.05\right)$, it produces this accurate solution using fewer particles. For the Mach 12 cylinder problem, method (iii) uses $65 \%$ of the particles required for method (i). Previous studies ${ }^{8,21}$ have demonstrated that if the computational time spent updating continuum NS regions is negligible compared the time spent updating DSMC regions, that the speedup achieved by the MPC method scales directly with the number of particles eliminated by NS modeling. However, the revised criteria for detecting continuum breakdown (method (iii)) requires significantly more NS updates. Instead of computing the $P_{B r}$ directly from the DSMC regions $\left(P_{B r}=K n_{G L}\right)$, a converged NS solution is required at each interface update for the sole purpose of comparing with DSMC $\left(P_{B r}=E_{Q}\right)$. Thus, the time spent on continuum regions is no longer negligible compared to that spent in DSMC regions. As a result, for the simulations considered in this article, method (iii) was found to take roughly the same computational time as method (i) despite using $65 \%$ of the particles. In addition to the added computational expense, method (iii) is sensitive to the level of statistical scatter in DSMC regions. The tolerance $(\varepsilon)$ must be set higher than the level of scatter, which for the blunt-body cylinder flows is approximately $1-2 \%$. If method (iii) is used with a tolerance below this level, statistical scatter is mistaken for a discrepancy between DSMC and NS solutions. Thus although robust for the simulations presented in this article, in general, method (iii) may be limited by statistical scatter and therefore not as robust as method (i).

\section{Conclusions}

This article presents a new method of determining continuum breakdown in hypersonic flows that is part of a modular particle-continuum (MPC) numerical method. The hybrid DSMC-NS method involves overlapping regions where both DSMC and NS methods are used. The new method of detecting continuum breakdown involves the direct comparison of DSMC and NS solutions within such overlapping regions. If the solutions agree within a user-specified tolerance, the interfaces are considered to be properly positioned in near-equilibrium regions. If the NS equations are unable to reproduce DSMC results in the overlap region, the interface locations are considered to lie in nonequilibrium regions and must be updated. Such a method of continuum breakdown detection eliminates the use of a breakdown parameter and associated empirical cutoff value used in the majority of prior hybrid DSMC-NS research.

The MPC method using this new criteria for continuum breakdown is shown to successfully reproduce full DSMC flowfield properties and heating rates with a high degree of accuracy for the hypersonic blunt-body flows studied in this article. The final interface locations between DSMC and NS regions are thus not reliant on typical continuum breakdown parameters and empirical cutoff values. Comparison between this method and the widely used gradientlength Knudsen number $\left(K n_{G L}\right)$ for continuum breakdown detection reveals that the $K n_{G L}$ parameter with a cutoff value of 0.05 is conservative in the forebody boundary layer and also conservative in the wake. This was verified since the new method requires fewer particles in the forebody and in the wake while still reproducing full DSMC results with equal accuracy. However, this new method of continuum breakdown detection is significantly more computationally demanding than simply computing the $K n_{G L}$ parameter and is also sensitive to statistical scatter in DSMC regions. As a result, it may not be a practical alternative for general incorporation within a hybrid DSMC-NS method. On the other hand, this study has shown that despite being conservative in some regions, the $K n_{G L}$ parameter (with a cutoff of 0.05 ) successfully predicts continuum breakdown and results in accurate hybrid solution results. Since computing the $K n_{G L}$ parameter is efficient and robust, the results of this article further support its general use in determining continuum breakdown as part of hybrid DSMC-NS simulations. 


\section{Acknowledgments}

The work performed at the University of Minnesota is supported by the Air Force Office of Scientific Research (AFOSR) under Grant No. FA9550-04-1-0341. The views and conclusions contained herein are those of the authors and should not be interpreted as necessarily representing the official polices or endorsements, either expressed or implied, of the AFOSR or the U.S. Government. The work performed at the University of Michigan is sponsored by the Constellation University Institutes Project through NASA grant NCC3-989.

\section{References}

${ }^{1}$ Hash, D. B. and Hassan, H. A., "Assessment of Schemes for Coupling Monte Carlo and Navier-Stokes Solution Methods," Journal of Thermophysics and Heat Transfer, Vol. 10, No. 2, 1996, pp. 242-249.

${ }^{2}$ Hash, D. B. and Hassan, H. A., "A Decoupled DSMC/Navier-Stokes Analysis of a Transitional Flow Experiment," AIAA Paper 96-0353, Jan. 1996, presented at the 34th AIAA Aerospace Sciences Meeting and Exhibit, Reno, NV.

${ }^{3}$ Roveda, R., Goldstein, D. B., and Varghese, P. L., "Hybrid Euler/Particle Approach for Continuum/Rarefied Flows," Journal of Spacecraft and Rockets, Vol. 35, No. 3, 1998, pp. 258-265.

${ }^{4}$ Roveda, R., Goldstein, D. B., and Varghese, P. L., "Hybrid Euler/Direct Simulation Monte Carlo Calculation of Unsteady Slit Flow," Journal of Spacecraft and Rockets, Vol. 37, No. 6, 2000, pp. 753-760.

${ }^{5}$ Wang, W. L. and Boyd, I. D., "Hybrid DSMC-CFD Simulations of Hypersonic Flow over Sharp and Blunted Bodies," AIAA Paper 03-3644, 2003, presented at the 36th AIAA Thermophysics Conference, Orlanda, Fl.

${ }^{6}$ Wijesinghe, H. S., Hornung, R. D., Garcia, A. L., and Hadjiconstantinou, N. G., "Three-dimensional Hybrid Continuum-Atomistic Simulations For Multiscale Hydrodynamics," Journal of Fluids Engineering, Vol. 126, 2004, pp. 768-777.

${ }^{7}$ Wu, J. S., Lian, Y. Y., Cheng, G., Koomullil, R. P., and Tseng, K. C., "Development and verification of a coupled DSMC-NS scheme using unstructured mesh," Journal of Computational Physics, Vol. 219, 2006, pp. 579-607.

${ }^{8}$ Schwartzentruber, T. E., Scalabrin, L., and Boyd, I. D., "A modular particle-continuum numerical method for hypersonic non-equilibrium gas flows," Journal of Computational Physics, Vol. 225, No. 1, 2007, pp. 1159-1174.

${ }^{9}$ Wijesinghe, H. S. and Hadjiconstantinou, N. G., "A Discussion of Hybrid Atomistic-Continuum Methods for Multiscale Hydrodynamics," International Journal for Multiscale Computational Engineering, Vol. 2, 2004.

${ }^{10}$ Schwartzentruber, T. E., Scalabrin, L. C., and Boyd, I. D., "Hybrid Particle-Continuum Simulations of Nonequilibrium Hypersonic BluntBody Flowfields," Journal of Thermophysics and Heat Transfer, Vol. 22, No. 1, 2008, pp. 29-37.

${ }^{11}$ Gallis, M. A., Torczynski, J. R., and Rader, D. J., "Molecular gas dynamics observations of Chapman-Enskog behavior and departures therefrom in nonequilibrium gases," Physical Review E, Vol. 69, 2004, pp. 42201-1 - 42201-4.

${ }^{12}$ Camberos, J. A., Schrock, C. R., McMullan, R. J., and Branam, R. D., "Development of Continuum Onset Criteria with Direct Simulation Monte-Carlo Using," AIAA Paper 06-2942, June 2006, presented at the 9th AIAA/ASME Joint Thermophysics and Heat Transfer Conference, San Francisco, CA.

${ }^{13}$ Boyd, I. D., Chen, G., and Candler, G. V., "Predicting Failure of the Continuum Fluid Equations in Transitional Hypersonic Flows," Physics of Fluids, Vol. 7, No. 1, 1995, pp. 210-219.

${ }^{14}$ Wang, W. L. and Boyd, I. D., "Predicting Continuum Breakdown in Hypersonic Viscous Flows," Physics of Fluids, Vol. 15, 2003 , pp. 91-100.

${ }^{15}$ Bird, G. A., Molecular Gas Dynamics and the Direct Simulation of Gas Flows, Oxford University Press, New York, 1994.

${ }^{16}$ Schwartzentruber, T. E. and Boyd, I. D., "A hybrid particle-continuum method applied to shock waves," Journal of Computational Physics, Vol. 215, No. 2, 2006, pp. 402-416.

${ }^{17}$ Schwartzentruber, T. E., Scalabrin, L. C., and Boyd, I. D., "Hybrid Particle-Continuum Simulations of Low Knudsen Number Hypersonic Flows," AIAA Paper 07-3829, June 2007, presented at the 39th AIAA Thermophysics Conference, Miami, FL.

${ }^{18}$ Dietrich, S. and Boyd, I. D., "Scalar and Parallel Optimized Implementation of the Direct Simulation Monte Carlo Method," Journal of Computational Physics, Vol. 126, 1996, pp. 328-342.

${ }^{19}$ Boyd, I. D., "Analysis of Rotational Nonequilibrium in Standing Shock Waves of Nitrogen," AIAA Journal, Vol. 28, No. 11, 1990, pp. 19971999.

${ }^{20}$ Scalabrin, L. C. and Boyd, I. D., "Numerical Simulation of Weakly Ionized Hypersonic Flow for Reentry Configurations," AIAA Paper 06-3773, 2006, presented at the 9th AIAA/ASME Joint Thermophysics and Heat Transfer Conference, San Francisco, CA.

${ }^{21}$ Schwartzentruber, T. E., Scalabrin, L. C., and Boyd, I. D., "Modular Implementation of a Hybrid DSMC-NS Solver for Hypersonic NonEquilibrium Flows," AIAA Paper 07-613, Jan. 2007, presented at the 45th AIAA Aerospace Sciences Meeting and Exhibit, Reno, NV.

${ }^{22}$ Garcia, A. L. and Alder, B. J., "Generation of the Chapman-Enskog Distribution," Journal of Computational Physics, Vol. 140, 1998, pp. 66-70.

${ }^{23}$ Sun, Q. and Boyd, I. D., "Evaluation of Macroscopic Properties in the Direct Simulation Monte Carlo Method," Journal of Thermophysics and Heat Transfer, Vol. 19, No. 3, 2005, pp. 329-335. 